Ann. Funct. Anal. 7 (2016), no. 2, 338-347

http://dx.doi.org/10.1215/20088752-3544417

ISSN: 2008-8752 (electronic)

ANNALS of

UNCTIONAL

http://projecteuclid.org/afa

ANALYSIS

\title{
CONE NONNEGATIVITY OF MOORE-PENROSE INVERSES OF UNBOUNDED GRAM OPERATORS
}

\author{
T. KURMAYYA ${ }^{1 *}$ and G. RAMESH ${ }^{2}$ \\ Communicated by M. Mbekhta
}

\begin{abstract}
In this article, necessary and sufficient conditions for the cone nonnegativity of Moore-Penrose inverses of unbounded Gram operators are derived. These conditions include statements on acuteness of certain closed convex cones in infinite-dimensional real Hilbert spaces.
\end{abstract}

\section{INTRODUCTION}

A real square matrix $T$ is called monotone if $x \geq 0$ whenever $T x \geq 0$. Here $x=\left(x_{i}\right) \geq 0$ means that $x_{i} \geq 0$ for all $i$. The concept of monotonicity was first proposed by Collatz [4] in connection with the application of finite difference methods for solving elliptic partial differential equations. He showed that a matrix is monotone if and only if it is invertible and the inverse is entrywise nonnegative; hence, the monotonicity of a matrix is equivalent to the nonnegativity of the inverse of a matrix.

The notion of monotonicity has been extended in a great variety of ways. We present a brief review here. Mangasarian [12] considered a rectangular matrix $T$ to be monotone if $T x \geq 0 \Rightarrow x \geq 0$. He showed, using the duality theorem of linear programming, that $T$ is monotone if and only if $T$ has a nonnegative left inverse. Berman and Plemmons generalized the concept of monotonicity in several ways in a series of articles, where they studied their relationships with nonnegativity of generalized inverses. The book by Berman and Plemmons [2] contains numerous examples of applications of nonnegative generalized inverses

Copyright 2016 by the Tusi Mathematical Research Group.

Received Sep. 21, 2015; Accepted Oct. 4, 2015.

${ }^{*}$ Corresponding author.

2010 Mathematics Subject Classification. Primary 15A09; Secondary 47H05, 15B48.

Keywords. Moore-Penrose inverse, unbounded Gram operator, cone, acute cone. 
Ann. Funct. Anal. 7 (2016), no. 2, 338-347

http://dx.doi.org/10.1215/20088752-3544417

ISSN: 2008-8752 (electronic)

ANNALS of

UNCTIONAL

http://projecteuclid.org/afa

ANALYSIS

\title{
CONE NONNEGATIVITY OF MOORE-PENROSE INVERSES OF UNBOUNDED GRAM OPERATORS
}

\author{
T. KURMAYYA ${ }^{1 *}$ and G. RAMESH ${ }^{2}$ \\ Communicated by M. Mbekhta
}

\begin{abstract}
In this article, necessary and sufficient conditions for the cone nonnegativity of Moore-Penrose inverses of unbounded Gram operators are derived. These conditions include statements on acuteness of certain closed convex cones in infinite-dimensional real Hilbert spaces.
\end{abstract}

\section{INTRODUCTION}

A real square matrix $T$ is called monotone if $x \geq 0$ whenever $T x \geq 0$. Here $x=\left(x_{i}\right) \geq 0$ means that $x_{i} \geq 0$ for all $i$. The concept of monotonicity was first proposed by Collatz [4] in connection with the application of finite difference methods for solving elliptic partial differential equations. He showed that a matrix is monotone if and only if it is invertible and the inverse is entrywise nonnegative; hence, the monotonicity of a matrix is equivalent to the nonnegativity of the inverse of a matrix.

The notion of monotonicity has been extended in a great variety of ways. We present a brief review here. Mangasarian [12] considered a rectangular matrix $T$ to be monotone if $T x \geq 0 \Rightarrow x \geq 0$. He showed, using the duality theorem of linear programming, that $T$ is monotone if and only if $T$ has a nonnegative left inverse. Berman and Plemmons generalized the concept of monotonicity in several ways in a series of articles, where they studied their relationships with nonnegativity of generalized inverses. The book by Berman and Plemmons [2] contains numerous examples of applications of nonnegative generalized inverses

Copyright 2016 by the Tusi Mathematical Research Group.

Received Sep. 21, 2015; Accepted Oct. 4, 2015.

${ }^{*}$ Corresponding author.

2010 Mathematics Subject Classification. Primary 15A09; Secondary 47H05, 15B48.

Keywords. Moore-Penrose inverse, unbounded Gram operator, cone, acute cone. 
Ann. Funct. Anal. 7 (2016), no. 2, 338-347

http://dx.doi.org/10.1215/20088752-3544417

ISSN: 2008-8752 (electronic)

ANNALS of

UNCTIONAL

http://projecteuclid.org/afa

ANALYSIS

\title{
CONE NONNEGATIVITY OF MOORE-PENROSE INVERSES OF UNBOUNDED GRAM OPERATORS
}

\author{
T. KURMAYYA ${ }^{1 *}$ and G. RAMESH ${ }^{2}$ \\ Communicated by M. Mbekhta
}

\begin{abstract}
In this article, necessary and sufficient conditions for the cone nonnegativity of Moore-Penrose inverses of unbounded Gram operators are derived. These conditions include statements on acuteness of certain closed convex cones in infinite-dimensional real Hilbert spaces.
\end{abstract}

\section{INTRODUCTION}

A real square matrix $T$ is called monotone if $x \geq 0$ whenever $T x \geq 0$. Here $x=\left(x_{i}\right) \geq 0$ means that $x_{i} \geq 0$ for all $i$. The concept of monotonicity was first proposed by Collatz [4] in connection with the application of finite difference methods for solving elliptic partial differential equations. He showed that a matrix is monotone if and only if it is invertible and the inverse is entrywise nonnegative; hence, the monotonicity of a matrix is equivalent to the nonnegativity of the inverse of a matrix.

The notion of monotonicity has been extended in a great variety of ways. We present a brief review here. Mangasarian [12] considered a rectangular matrix $T$ to be monotone if $T x \geq 0 \Rightarrow x \geq 0$. He showed, using the duality theorem of linear programming, that $T$ is monotone if and only if $T$ has a nonnegative left inverse. Berman and Plemmons generalized the concept of monotonicity in several ways in a series of articles, where they studied their relationships with nonnegativity of generalized inverses. The book by Berman and Plemmons [2] contains numerous examples of applications of nonnegative generalized inverses

Copyright 2016 by the Tusi Mathematical Research Group.

Received Sep. 21, 2015; Accepted Oct. 4, 2015.

${ }^{*}$ Corresponding author.

2010 Mathematics Subject Classification. Primary 15A09; Secondary 47H05, 15B48.

Keywords. Moore-Penrose inverse, unbounded Gram operator, cone, acute cone. 
Ann. Funct. Anal. 7 (2016), no. 2, 338-347

http://dx.doi.org/10.1215/20088752-3544417

ISSN: 2008-8752 (electronic)

ANNALS of

UNCTIONAL

http://projecteuclid.org/afa

ANALYSIS

\title{
CONE NONNEGATIVITY OF MOORE-PENROSE INVERSES OF UNBOUNDED GRAM OPERATORS
}

\author{
T. KURMAYYA ${ }^{1 *}$ and G. RAMESH ${ }^{2}$ \\ Communicated by M. Mbekhta
}

\begin{abstract}
In this article, necessary and sufficient conditions for the cone nonnegativity of Moore-Penrose inverses of unbounded Gram operators are derived. These conditions include statements on acuteness of certain closed convex cones in infinite-dimensional real Hilbert spaces.
\end{abstract}

\section{INTRODUCTION}

A real square matrix $T$ is called monotone if $x \geq 0$ whenever $T x \geq 0$. Here $x=\left(x_{i}\right) \geq 0$ means that $x_{i} \geq 0$ for all $i$. The concept of monotonicity was first proposed by Collatz [4] in connection with the application of finite difference methods for solving elliptic partial differential equations. He showed that a matrix is monotone if and only if it is invertible and the inverse is entrywise nonnegative; hence, the monotonicity of a matrix is equivalent to the nonnegativity of the inverse of a matrix.

The notion of monotonicity has been extended in a great variety of ways. We present a brief review here. Mangasarian [12] considered a rectangular matrix $T$ to be monotone if $T x \geq 0 \Rightarrow x \geq 0$. He showed, using the duality theorem of linear programming, that $T$ is monotone if and only if $T$ has a nonnegative left inverse. Berman and Plemmons generalized the concept of monotonicity in several ways in a series of articles, where they studied their relationships with nonnegativity of generalized inverses. The book by Berman and Plemmons [2] contains numerous examples of applications of nonnegative generalized inverses

Copyright 2016 by the Tusi Mathematical Research Group.

Received Sep. 21, 2015; Accepted Oct. 4, 2015.

${ }^{*}$ Corresponding author.

2010 Mathematics Subject Classification. Primary 15A09; Secondary 47H05, 15B48.

Keywords. Moore-Penrose inverse, unbounded Gram operator, cone, acute cone. 
Ann. Funct. Anal. 7 (2016), no. 2, 338-347

http://dx.doi.org/10.1215/20088752-3544417

ISSN: 2008-8752 (electronic)

ANNALS of

UNCTIONAL

http://projecteuclid.org/afa

ANALYSIS

\title{
CONE NONNEGATIVITY OF MOORE-PENROSE INVERSES OF UNBOUNDED GRAM OPERATORS
}

\author{
T. KURMAYYA ${ }^{1 *}$ and G. RAMESH ${ }^{2}$ \\ Communicated by M. Mbekhta
}

\begin{abstract}
In this article, necessary and sufficient conditions for the cone nonnegativity of Moore-Penrose inverses of unbounded Gram operators are derived. These conditions include statements on acuteness of certain closed convex cones in infinite-dimensional real Hilbert spaces.
\end{abstract}

\section{INTRODUCTION}

A real square matrix $T$ is called monotone if $x \geq 0$ whenever $T x \geq 0$. Here $x=\left(x_{i}\right) \geq 0$ means that $x_{i} \geq 0$ for all $i$. The concept of monotonicity was first proposed by Collatz [4] in connection with the application of finite difference methods for solving elliptic partial differential equations. He showed that a matrix is monotone if and only if it is invertible and the inverse is entrywise nonnegative; hence, the monotonicity of a matrix is equivalent to the nonnegativity of the inverse of a matrix.

The notion of monotonicity has been extended in a great variety of ways. We present a brief review here. Mangasarian [12] considered a rectangular matrix $T$ to be monotone if $T x \geq 0 \Rightarrow x \geq 0$. He showed, using the duality theorem of linear programming, that $T$ is monotone if and only if $T$ has a nonnegative left inverse. Berman and Plemmons generalized the concept of monotonicity in several ways in a series of articles, where they studied their relationships with nonnegativity of generalized inverses. The book by Berman and Plemmons [2] contains numerous examples of applications of nonnegative generalized inverses

Copyright 2016 by the Tusi Mathematical Research Group.

Received Sep. 21, 2015; Accepted Oct. 4, 2015.

${ }^{*}$ Corresponding author.

2010 Mathematics Subject Classification. Primary 15A09; Secondary 47H05, 15B48.

Keywords. Moore-Penrose inverse, unbounded Gram operator, cone, acute cone. 
Ann. Funct. Anal. 7 (2016), no. 2, 338-347

http://dx.doi.org/10.1215/20088752-3544417

ISSN: 2008-8752 (electronic)

ANNALS of

UNCTIONAL

http://projecteuclid.org/afa

ANALYSIS

\title{
CONE NONNEGATIVITY OF MOORE-PENROSE INVERSES OF UNBOUNDED GRAM OPERATORS
}

\author{
T. KURMAYYA ${ }^{1 *}$ and G. RAMESH ${ }^{2}$ \\ Communicated by M. Mbekhta
}

\begin{abstract}
In this article, necessary and sufficient conditions for the cone nonnegativity of Moore-Penrose inverses of unbounded Gram operators are derived. These conditions include statements on acuteness of certain closed convex cones in infinite-dimensional real Hilbert spaces.
\end{abstract}

\section{INTRODUCTION}

A real square matrix $T$ is called monotone if $x \geq 0$ whenever $T x \geq 0$. Here $x=\left(x_{i}\right) \geq 0$ means that $x_{i} \geq 0$ for all $i$. The concept of monotonicity was first proposed by Collatz [4] in connection with the application of finite difference methods for solving elliptic partial differential equations. He showed that a matrix is monotone if and only if it is invertible and the inverse is entrywise nonnegative; hence, the monotonicity of a matrix is equivalent to the nonnegativity of the inverse of a matrix.

The notion of monotonicity has been extended in a great variety of ways. We present a brief review here. Mangasarian [12] considered a rectangular matrix $T$ to be monotone if $T x \geq 0 \Rightarrow x \geq 0$. He showed, using the duality theorem of linear programming, that $T$ is monotone if and only if $T$ has a nonnegative left inverse. Berman and Plemmons generalized the concept of monotonicity in several ways in a series of articles, where they studied their relationships with nonnegativity of generalized inverses. The book by Berman and Plemmons [2] contains numerous examples of applications of nonnegative generalized inverses

Copyright 2016 by the Tusi Mathematical Research Group.

Received Sep. 21, 2015; Accepted Oct. 4, 2015.

${ }^{*}$ Corresponding author.

2010 Mathematics Subject Classification. Primary 15A09; Secondary 47H05, 15B48.

Keywords. Moore-Penrose inverse, unbounded Gram operator, cone, acute cone. 
Ann. Funct. Anal. 7 (2016), no. 2, 338-347

http://dx.doi.org/10.1215/20088752-3544417

ISSN: 2008-8752 (electronic)

ANNALS of

UNCTIONAL

http://projecteuclid.org/afa

ANALYSIS

\title{
CONE NONNEGATIVITY OF MOORE-PENROSE INVERSES OF UNBOUNDED GRAM OPERATORS
}

\author{
T. KURMAYYA ${ }^{1 *}$ and G. RAMESH ${ }^{2}$ \\ Communicated by M. Mbekhta
}

\begin{abstract}
In this article, necessary and sufficient conditions for the cone nonnegativity of Moore-Penrose inverses of unbounded Gram operators are derived. These conditions include statements on acuteness of certain closed convex cones in infinite-dimensional real Hilbert spaces.
\end{abstract}

\section{INTRODUCTION}

A real square matrix $T$ is called monotone if $x \geq 0$ whenever $T x \geq 0$. Here $x=\left(x_{i}\right) \geq 0$ means that $x_{i} \geq 0$ for all $i$. The concept of monotonicity was first proposed by Collatz [4] in connection with the application of finite difference methods for solving elliptic partial differential equations. He showed that a matrix is monotone if and only if it is invertible and the inverse is entrywise nonnegative; hence, the monotonicity of a matrix is equivalent to the nonnegativity of the inverse of a matrix.

The notion of monotonicity has been extended in a great variety of ways. We present a brief review here. Mangasarian [12] considered a rectangular matrix $T$ to be monotone if $T x \geq 0 \Rightarrow x \geq 0$. He showed, using the duality theorem of linear programming, that $T$ is monotone if and only if $T$ has a nonnegative left inverse. Berman and Plemmons generalized the concept of monotonicity in several ways in a series of articles, where they studied their relationships with nonnegativity of generalized inverses. The book by Berman and Plemmons [2] contains numerous examples of applications of nonnegative generalized inverses

Copyright 2016 by the Tusi Mathematical Research Group.

Received Sep. 21, 2015; Accepted Oct. 4, 2015.

${ }^{*}$ Corresponding author.

2010 Mathematics Subject Classification. Primary 15A09; Secondary 47H05, 15B48.

Keywords. Moore-Penrose inverse, unbounded Gram operator, cone, acute cone. 
Ann. Funct. Anal. 7 (2016), no. 2, 338-347

http://dx.doi.org/10.1215/20088752-3544417

ISSN: 2008-8752 (electronic)

ANNALS of

UNCTIONAL

http://projecteuclid.org/afa

ANALYSIS

\title{
CONE NONNEGATIVITY OF MOORE-PENROSE INVERSES OF UNBOUNDED GRAM OPERATORS
}

\author{
T. KURMAYYA ${ }^{1 *}$ and G. RAMESH ${ }^{2}$ \\ Communicated by M. Mbekhta
}

\begin{abstract}
In this article, necessary and sufficient conditions for the cone nonnegativity of Moore-Penrose inverses of unbounded Gram operators are derived. These conditions include statements on acuteness of certain closed convex cones in infinite-dimensional real Hilbert spaces.
\end{abstract}

\section{INTRODUCTION}

A real square matrix $T$ is called monotone if $x \geq 0$ whenever $T x \geq 0$. Here $x=\left(x_{i}\right) \geq 0$ means that $x_{i} \geq 0$ for all $i$. The concept of monotonicity was first proposed by Collatz [4] in connection with the application of finite difference methods for solving elliptic partial differential equations. He showed that a matrix is monotone if and only if it is invertible and the inverse is entrywise nonnegative; hence, the monotonicity of a matrix is equivalent to the nonnegativity of the inverse of a matrix.

The notion of monotonicity has been extended in a great variety of ways. We present a brief review here. Mangasarian [12] considered a rectangular matrix $T$ to be monotone if $T x \geq 0 \Rightarrow x \geq 0$. He showed, using the duality theorem of linear programming, that $T$ is monotone if and only if $T$ has a nonnegative left inverse. Berman and Plemmons generalized the concept of monotonicity in several ways in a series of articles, where they studied their relationships with nonnegativity of generalized inverses. The book by Berman and Plemmons [2] contains numerous examples of applications of nonnegative generalized inverses

Copyright 2016 by the Tusi Mathematical Research Group.

Received Sep. 21, 2015; Accepted Oct. 4, 2015.

${ }^{*}$ Corresponding author.

2010 Mathematics Subject Classification. Primary 15A09; Secondary 47H05, 15B48.

Keywords. Moore-Penrose inverse, unbounded Gram operator, cone, acute cone. 
Ann. Funct. Anal. 7 (2016), no. 2, 338-347

http://dx.doi.org/10.1215/20088752-3544417

ISSN: 2008-8752 (electronic)

ANNALS of

UNCTIONAL

http://projecteuclid.org/afa

ANALYSIS

\title{
CONE NONNEGATIVITY OF MOORE-PENROSE INVERSES OF UNBOUNDED GRAM OPERATORS
}

\author{
T. KURMAYYA ${ }^{1 *}$ and G. RAMESH ${ }^{2}$ \\ Communicated by M. Mbekhta
}

\begin{abstract}
In this article, necessary and sufficient conditions for the cone nonnegativity of Moore-Penrose inverses of unbounded Gram operators are derived. These conditions include statements on acuteness of certain closed convex cones in infinite-dimensional real Hilbert spaces.
\end{abstract}

\section{INTRODUCTION}

A real square matrix $T$ is called monotone if $x \geq 0$ whenever $T x \geq 0$. Here $x=\left(x_{i}\right) \geq 0$ means that $x_{i} \geq 0$ for all $i$. The concept of monotonicity was first proposed by Collatz [4] in connection with the application of finite difference methods for solving elliptic partial differential equations. He showed that a matrix is monotone if and only if it is invertible and the inverse is entrywise nonnegative; hence, the monotonicity of a matrix is equivalent to the nonnegativity of the inverse of a matrix.

The notion of monotonicity has been extended in a great variety of ways. We present a brief review here. Mangasarian [12] considered a rectangular matrix $T$ to be monotone if $T x \geq 0 \Rightarrow x \geq 0$. He showed, using the duality theorem of linear programming, that $T$ is monotone if and only if $T$ has a nonnegative left inverse. Berman and Plemmons generalized the concept of monotonicity in several ways in a series of articles, where they studied their relationships with nonnegativity of generalized inverses. The book by Berman and Plemmons [2] contains numerous examples of applications of nonnegative generalized inverses

Copyright 2016 by the Tusi Mathematical Research Group.

Received Sep. 21, 2015; Accepted Oct. 4, 2015.

${ }^{*}$ Corresponding author.

2010 Mathematics Subject Classification. Primary 15A09; Secondary 47H05, 15B48.

Keywords. Moore-Penrose inverse, unbounded Gram operator, cone, acute cone. 
Ann. Funct. Anal. 7 (2016), no. 2, 338-347

http://dx.doi.org/10.1215/20088752-3544417

ISSN: 2008-8752 (electronic)

ANNALS of

UNCTIONAL

http://projecteuclid.org/afa

ANALYSIS

\title{
CONE NONNEGATIVITY OF MOORE-PENROSE INVERSES OF UNBOUNDED GRAM OPERATORS
}

\author{
T. KURMAYYA ${ }^{1 *}$ and G. RAMESH ${ }^{2}$ \\ Communicated by M. Mbekhta
}

\begin{abstract}
In this article, necessary and sufficient conditions for the cone nonnegativity of Moore-Penrose inverses of unbounded Gram operators are derived. These conditions include statements on acuteness of certain closed convex cones in infinite-dimensional real Hilbert spaces.
\end{abstract}

\section{INTRODUCTION}

A real square matrix $T$ is called monotone if $x \geq 0$ whenever $T x \geq 0$. Here $x=\left(x_{i}\right) \geq 0$ means that $x_{i} \geq 0$ for all $i$. The concept of monotonicity was first proposed by Collatz [4] in connection with the application of finite difference methods for solving elliptic partial differential equations. He showed that a matrix is monotone if and only if it is invertible and the inverse is entrywise nonnegative; hence, the monotonicity of a matrix is equivalent to the nonnegativity of the inverse of a matrix.

The notion of monotonicity has been extended in a great variety of ways. We present a brief review here. Mangasarian [12] considered a rectangular matrix $T$ to be monotone if $T x \geq 0 \Rightarrow x \geq 0$. He showed, using the duality theorem of linear programming, that $T$ is monotone if and only if $T$ has a nonnegative left inverse. Berman and Plemmons generalized the concept of monotonicity in several ways in a series of articles, where they studied their relationships with nonnegativity of generalized inverses. The book by Berman and Plemmons [2] contains numerous examples of applications of nonnegative generalized inverses

Copyright 2016 by the Tusi Mathematical Research Group.

Received Sep. 21, 2015; Accepted Oct. 4, 2015.

${ }^{*}$ Corresponding author.

2010 Mathematics Subject Classification. Primary 15A09; Secondary 47H05, 15B48.

Keywords. Moore-Penrose inverse, unbounded Gram operator, cone, acute cone. 